\title{
Biomarkers of the brain injuries - the future diagnosis standard in head trauma? Brief literature review
}

\author{
Andreea Elena Bîrlescu ${ }^{1,2}$, Bianca Hanganu ${ }^{1}$, \\ Andreea Alexandra Hleșcu ${ }^{1,2}$, Irina Smaranda \\ Manoilescu ${ }^{1,2}$, Beatrice Gabriela loan ${ }^{1,2}$ \\ 1 "Grigore T. Popa" University of Medicine and Pharmacy, Dept. of \\ Legal Medicine, Iași, ROMANIA \\ 2 Institute of Legal Medicine, Iasi, RomanIA
}

\begin{abstract}
Acute head trauma is often a clinical challenge in diagnosing the brain damage, assessing its severity and prognosis, and establishing the optimal treatment. Different patients, with brain damage of apparent comparable severity according to the imaging examination, may have different neurological evolution or different response to therapy.

Minor traumatic brain injuries can induce a brief loss of consciousness or confusion, are usually benign, but sometimes they cause persistent and progressive brain symptoms in the long run. However, at present, there are no reliable methods that can diagnose properly minor traumatic brain injuries.

Biomarkers of the brain injuries allow the monitoring of both physiological and pathological processes. The identification of such biomarkers could allow a better understanding of the pathological processes involved in traumatic brain injuries, their diagnosis, prognosis and may facilitate the establishment of a better treatment regimen for these patients.

In this article, the authors make a brief review of the literature in which they analyse the biomarkers of the lesions of the various brain structures identified so far, which can be detected in biological fluids (blood, cerebrospinal fluid) and the advantages and limitations of their use in the current medical practice.
\end{abstract}

\section{INTRODUCTION}

Brain injury may be graded by Glasgow Coma Scale score (GCS) in: severe trauma, characterized by a GCS score of less than or equal to 8the cerebral coma equivalent; moderate trauma, characterized by a GCS score of 9-12 [1], of which $10 \%$ of patients will experience neurological deterioration and cerebral coma and minor trauma, where the GCS score is between 13 and 15 [2].

Minor brain trauma can be characterized by the loss of consciousness of short duration- up to 30 minutes, or confusion, retrograde amnesia to the traumatic event of up to 24 hours, headache,

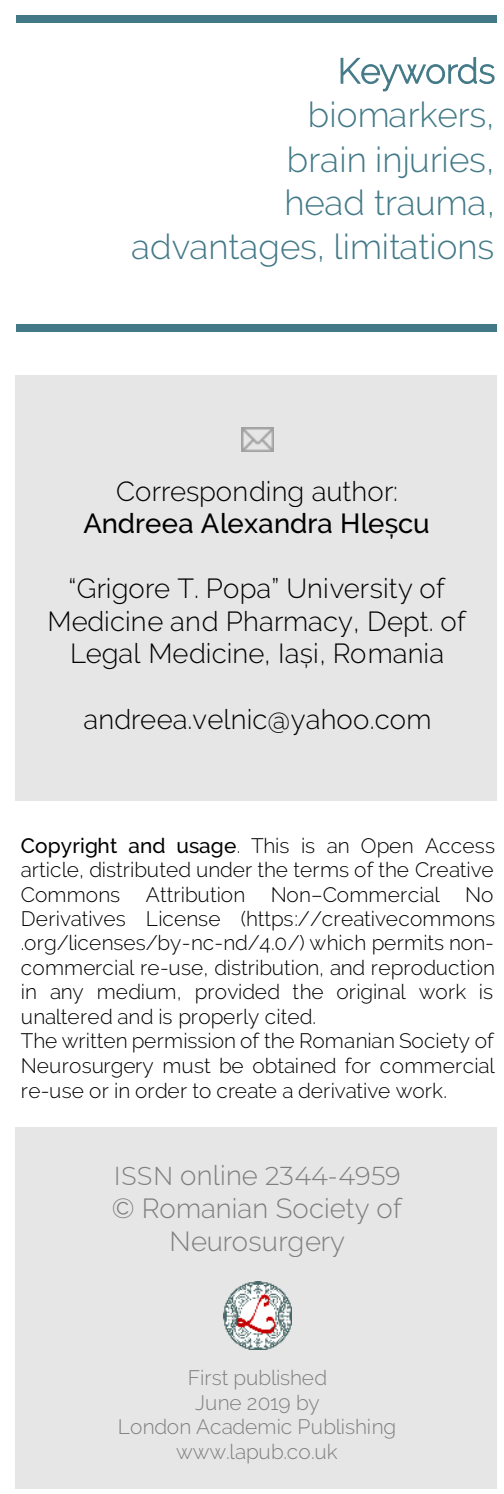


vomiting (unrelated to intracranial hypertension) and/ or transient focal neurological signs or convulsions [1]. Most patients with minor brain trauma show favourable progression, but about 3\% of cases have an unfavourable progression, with increased risk for intracranial haemorrhage and diffuse axonal injuries, the promoters of cognitive, motor and psychosocial deficits [2]. The morbidity associated with cerebral traumatic injuries (even minor) is considerable. Studies have shown that between 1 and $20 \%$ of patients with minor traumatic brain injuries develop persistent physical, cognitive and behavioural disorders [3], such as chronic dizziness, fatigue, headache, and amnesia. It is also important that in the clinic, minor brain injuries are more common than stroke, dementia and epilepsy, indicating their high prevalence and justifying the efforts to diagnose and treat them as accurately as possible.

Despite substantial efforts to clarify and improve the diagnostic criteria for minor traumatic brain injuries, compared to moderate and severe brain injuries, the former often remain a diagnostic challenge. This is largely due to the rapid resolution of acute signs and symptoms after a simple rest and the absence, in many cases, of objective neuroimaging evidence.

Current diagnosis regimens for minor traumatic brain injuries often face the difficulty of differentiating them from non-traumatic pathologies that may exhibit a similar symptomatology.

Currently, the gold standard for diagnosing and establishing the therapeutic management of traumatic brain injuries is the computer tomography (CT) exam. It allows the detection of various traumatic head injuries, such as cranial fractures, extra- and subdural hematomas, subarachnoid haemorrhage, cerebral contusion and laceration, cerebral edema, etc. With increased sensitivity and specificity and by using it in the clinic as a routine exam, the head CT scan surpassed the simple head radiography [1]. However, the head radiography retains its importance in the initial classification of traumatic brain injuries as complicated injuries (radiographically proven) or uncomplicated injuries (negative radiography), and thus contributes to establishing the necessary further investigations, such as CT scan or MRI, and the therapeutic management (hospital admission with or without surgery). Despite the superior results from classical
X-rays, modern imaging modalities such as CT scan and MRI are costly and entail a number of risks, including the risk of irradiation and the risk associated with the administration of the contrast substance [3]. Also, in many cases, minor traumatic brain injuries cannot be detected by CT scan. Under these circumstances, an additional diagnostic tool is necessary to detect patients at risk of developing further complications.

\section{BIOMARKERS OF CEREBRAL LESIONS -}

\section{PATHOPHYSIOLOGICAL BACKGROUND}

Research on biomarkers of neuronal lesions began after the 1950s, and their interest has increased significantly over the past 25 years.

Biomarkers, also called biological markers, are natural characteristics that can be measured and interpreted objectively as indicators of biological processes or responses to therapeutic interventions [4]. Biomarkers are indicators of physiological, pathological or pharmacological processes. Each organ system has more or less specific biomarkers, and their analysis, either isolated or joined to other clinical investigations, allows monitoring of an individual's health status [2].

From the pathophysiological point of view, due to the brain injuries, the neuronal and astroglial network loses its structural integrity, cellular membranes are affected and secondary to these events, biomarkers are released in the cerebrospinal fluid and in blood, allowing for the diagnosis and prognosis of brain injuries [5].

Traumatic mechanical forces can determine cell damage due to shear, rupture and stretching of neurons, axons, glial cells and blood vessels, and the lesion will induce biochemical changes such as excitotoxicity, necrosis and apoptosis, oxidative stress and inflammation. Similar pathophysiological changes can also be seen in disorders induced by acute pathological brain injury such as stroke.

Sensitive and specific biomarkers that reflect the brain damage can provide important information about the pathophysiology of traumatic brain injuries and can predict abnormal CT results and/ or the development of residual deficits in patients suffering from minor traumatic brain injuries. Biomarkers could be diagnostic criteria for traumatic brain injuries and could be a valuable adjuvant to clinical and routine imaging. In particular, the possibility of using biomarkers in patients with minor 
traumatic brain injuries could provide a rapid, differential, non-invasive and cost-effective diagnostic test to guide appropriate patients' triage and their early management [6].

\section{BIOMARKERS OF BRAIN INJURIES -}

PROMISING RESULTS

A wide range of proteins, of different origins and resulting from various pathways, have been studied as biomarkers for diagnosis and prognosis of brain injuries. However, the performance of many of these biomarkers has not been studied in the case of minor traumatic brain injuries [7].

At the level of the central nervous system, the lesion biomarkers studied to date are S100beta, Glial Fibrillary Acidic Protein (GFAP), Neuron- Specific Enolase (NSE), Alpha II Spectrin, Tau protein, Ubiquitin C-Terminal Hydrolase L1 (UCH-L1), FattyAcid-Binding Proteins (B-FABP, H-FABP) and II-10.

Among the listed biomarkers, the most studied are S100 beta and Neuron- Specific Enolase (NSE), the values of which increase in hypoxic conditions, starting on the 2nd post-traumatic day and normalize at about 4 days after the trauma [2].

5100 beta is a dimer that binds cell calcium, is involved in cellular differentiation and neuronal proliferation and has a life span of about 2 hours. There are 19 types of such dimers, of which S100A1 (in skeletal muscles, heart, and kidneys), S100A1B (in astrocytes), S100B (in astrocytes and Schwan cells) and S100BB (in astrocytes). The low molecular weight of $21 \mathrm{kDa}$ allows the $\$ 100$ beta dimers to easily cross the blood-brain barrier, so that in brain injuries high levels of S100beta are found in the blood. Unlike the NSE, the plasma level of S100beta is not affected by hemolysis, 21.2 micrograms/ liter suggesting the installation of anoxic coma, and 15.2 micrograms/ liter indicates neuronal recovery.

Neuron-Specific Enolase (NSE) is an isoform dimer involved in glucose metabolism, which is normally not found in the peripheral blood. In patients with stroke, the NSE value increases, with higher values for patients with irrecoverable traumatic brain injuries compared to patients with favourable progression. Decreasing NSE values at 24-48 hours after the trauma usually indicates a good prognosis, while a value greater than or equal to 30 micrograms/ liter, 48 hours post-trauma, predicted death in $100 \%$ of the cases. As mentioned above, NSE values are influenced by hemolysis, which does not allow its determination in peripheral blood. Apart from brain lesions, other sources of NSE may also be small cell carcinomas, neuroblastoma, haemorrhagic shock, femoral fracture, ischemia and local reperfusion.

Glial Fibrillary Acidic Protein (GFAP) is a monomer, being an intermediate protein derived from astroglial cells. GFAP has increased specificity for neuronal tissue, with high values in degenerative brain diseases, cerebral infarction, severe brain injury, and axonal injuries. GFAP is a predictive indicator for the recovery of anoxic cerebral coma, but studies conducted so far on this monomer are contradictory, and further research is needed on post-mortem biological products.

Ubiquitin C-Terminal Hydrolase L1 (UCH-L1) is a compound that plays a role in the elimination of oxidized neuronal proteins under both normal and pathological conditions. Initially, it was used as a histological marker for neurons. Recently, UCH-L1 has been found to have elevated values in the cerebrospinal fluid after a traumatic brain injury, which can be immediately detected posttraumatically, with elevated values lasting for about one week [2].

Both S100B and the combination of GFAP and UCH-L1 were promising in screening for CT positivity/ negativity among patients with acute traumatic brain injury [8].

Alpha II Spectrin is a major component of the cortical membrane of the cytoskeleton, being present in axons and presynaptic terminations. It is a marker for apoptosis and necrosis in the posttraumatic initial stages and has high values in moderate and severe brain injuries [2].

Tau is a microtubule associated protein, which is necessary to maintain the structural integrity of the axons. Tau proteins have also other functions, such as nerve impulse transmission, synaptic activity, cellular proliferation, neurobiological development and neuroplasticity. Phosphorylation of Tau proteins is a normal metabolic process, while in both aging and neurodegenerative diseases, Tau proteins undergo hyper-phosphorylation, which determines their aggregation as fibrillar deposits. Post mortem studies on human corpses described different patterns of taunting, depending on the pathological phenotype. Recent studies also highlight the uniqueness of pathological models, including a model attributed to repetitive cerebral trauma, 
although clinical correlations were relative [9].

Fatty-Acid-Binding Proteins (FABPS) are nonenzymatic cytoplasmic proteins involved in intracellular buffering and transport of fatty acids. These are 9 distinct protein types, each named after the tissue in which it was first detected. FABPs are rapidly released into circulation from the injured cells and are eliminated by the kidney, with a half-life of about 20 minutes. B-FABP was first identified in the rodent brain where it has a variable concentration depending on the animal's age (stage of development). Thus, in adult mice, B-FABP is usually produced at low concentrations and is detected only in glial cells of the white matter. Unlike $B-F A B P, H-F A B P$ is also detected in neurons of the gray matter. B-FABP and H-FABP proteins have different brain tissue distribution, with the highest concentrations in the frontal lobe. However, in all brain structures it was observed that the level of $\mathrm{H}$ FABP concentration is about 10 times higher than the B-FABP concentration. Studies show that these two proteins have greater susceptibility to minor cerebral lesions than the currently used markers, S100B and NSE respectively [10].

DIAGNOSTIC RELEVANCE OF BIOMARKERS OF CEREBRAL INJURIES

To date, biomarkers of cerebral injuries have been detected in cerebrospinal fluid and in peripheral blood. It has been found that in cases where the blood-brain barrier is intact, cerebral proteins are only present in small amounts in blood. The condition of the blood-brain barrier has, therefore, an important influence on the concentration of those proteins in the blood, which should be considered for the interpretation of the cerebral lesion-specific biomarkers [7].

The cerebrospinal fluid is in direct contact with the extracellular matrix of the brain, and its composition reflects the biochemical changes occurring in this organ. For these reasons, the cerebrospinal fluid could be an optimal source of brain damage biomarkers. Several cerebral lesionspecific biomarkers have already been described, including proteins that indicate the integrity of the blood-brain barrier and neuro-inflammation, as well as axonal, neuronal and astrogial lesions. Some proteins that are expressed in the central nervous system are also detectable in peripheral blood, albeit at very low concentrations due to their dilution in the much larger volume of the extracellular plasma and matrix of peripheral tissues. Because peripheral blood sampling is much easier in practice than the collection of the cerebrospinal fluid, a series of cerebrospinal fluid biomarkers specific for minor traumatic brain injuries have also been evaluated in the peripheral blood. The low concentration of potential biomarkers in the peripheral blood is, however, a technical limitation on the use of most standard immunological tests. However, the number of potential biomarkers of cerebral lesions in the peripheral blood studied is steadily increasing as the analytical tools for detecting them become more and more sensitive [11].

Studies have shown that unique biomarkers do not have the specificity and sensitivity required for their use as diagnostic tools. For a biomarker to be useful its sensitivity and specificity should be very high to ensure diagnosis and prognosis assessment without the need for a CT brain exam.

So far, most research on biomarkers of minor traumatic brain injuries has been performed with unique biomarkers. The combination of different biomarkers has been suggested to enhance the diagnostic performance. Several studies have shown that combinations of biomarkers significantly increase diagnostic performance in various pathologies, such as sleep disorders, post-stroke subarachnoid haemorrhage, lung cancer or differentiation of post-traumatic brain injuries from other types of lesions. Furthermore, it has been suggested that some combinations of different clinical parameters, such as the age and types of biomarkers, e.g. inflammatory proteins, can improve the classification of lesions [7].

In a multicentre study, 13 cerebral biomarkers, all previously investigated in patients with stroke, were evaluated for their ability to correctly classify patients with minor traumatic brain injury, CT positive and CT negative, with a GCS score of 15 and showing at least one clinical symptom. Of the 13 biomarkers, the $\mathrm{H}$ FABP and IL 10 proteins were the best single markers. These were further compared and combined with the better-studied S100B and GFAP markers. H-FABP was the best single marker, but when combined with GFAP, the overall performance increased from $32 \%$ to $46 \%$, with a sensitivity of $100 \%$. Proteins have been shown to be released from various types of injured cells. S100B and GFAP 
were derived from astrocytic lesions, H-FABP from endothelial cells and neuronal cellular bodies, while IL10 is expressed by monocytes and macrophages [7].

Detection of cerebral lesions by the serum biomarkers is not a standard procedure in current clinical practice, although several proteins, such as S100B, NSE, myelin basic protein and GFAP show promising results [10]. Some biomarkers, such as S100B and GFAP, have been extensively studied in the blood of patients with minor traumatic brain injuries, but so far none seem to provide sufficient information [7].

\section{DIFFICULTIES AND LIMITATIONS IN THE STUDY OF BIOMARKERS OF BRAIN INJURIES}

The main difficulty facing biomarkers for brain damage is to know whether the measured proteins really come from the brain injuries. As shown above, regardless of their origin, single biomarkers do not have sufficient performance to be transformed into diagnostic tools. Biomarker combinations, however, have been shown to enhance diagnostic performance when proteins of different origins and pathways are combined, due to the complexity of the nervous system and the heterogeneity of the traumatic brain injuries $[7,8]$.

There are also other obstacles to the development of a series of blood biomarkers for minor traumatic brain injuries. The blood-brain barrier prevents the evaluation of the biochemical changes in the brain by using biomarkers in the blood, but this is possible, however, in the case of loss of blood-brain barrier integrity, which occurs in severe brain lesions. In addition, some potential biomarkers suffer a proteolytic degradation in the blood, and their levels may be affected by clearance in the blood through the liver or kidneys. The accuracy of immunoassays may also be affected by the binding of biomarkers to carrier proteins and extra-cerebral sources of biomarkers [12].

Biomarkers of the cerebral injuries have different delivery patterns, and this has limitations on their practical use. As a result, clinical applicability may be limited by the type of brain injury (traumatic, stroke, hypoxia-ischemia). Another important limitation in the analysis of cerebral biomarkers is their ambiguity in multiple lesions.
Despite the current limitations in the study and application of biomarkers of cerebral lesions in the current medical practice, biomarkers could be used in the future as an adjuvant, supplementing the traditional and neuro-imaging examination in the diagnosis and prognosis of patients with traumatic brain injuries [4].

\section{CONCLUSIONS}

Traumatic brain injuries may raise clinical challenges due to the diagnostic difficulties and the lack of specific prognostic tools. A special place in the traumatic pathology of the brain is occupied by minor traumatic brain injuries that, although characterized by immediate mild signs and symptoms, can induce long-term brain pathology with increased disability potential.

Biomarkers of cerebral injuries may be a new diagnostic standard for traumatic brain injuries, and in particular, minor ones that often cannot be detected by cerebral CT.

However, further studies are needed to identify the biomarkers or combinations of biomarkers with the highest sensitivity and specificity for cerebral injuries.

\section{REFERENCES}

1. Popescu I, Florian IS, Poeată I. Tratat de neurochirurgie. București: Editura Academiei Române, 2014.

2. Chirica VI. Useful markers to assess traumatic and hypoxic brain injury. Rom J Leg Med. 2017; 25: 146-151.

3. Sharma R, Rosenberg A, Bennet ER, Laskowitz DT, Acheson SK. A blood-based biomarker panel to risk stratify in mild traumatic brain injury. PLoS One. 2017; 12(3): e0173798.

4. Toman E, Harrisson S, Belli T. Biomarkers in traumatic brain injury: a review. Journal of the Royal Army Medical Corps. 2016; 162(2):103-108.

5. Mondello S, Schmid K, Berger RP, Kobeissy F, Jeromin A, Italiano $D$, Buki $A$. The challenge of mild traumatic brain injury: role of biochemical markers in diagnosis of brain damage. Med Res Rev. 2014 May; 34(3):503-531.

6. http://www.traumaticbraininjury.com/understandingtbi/what-are-the-causes-of-tbi/

7. Mrozek S, Dumurgier J, Citerio G, Mebazaa A, Geeraerts T. Biomarkers and acute brain injury: interest and limits. Crit Care. 2014; 18:220.

8. Pelsers MMAL, Hanhoff T, Van Der Voort D et all. Brain and heart type fatty acid binding proteins in the brain: tissue distribution and clinical utility. Clin Chem. 2004; 50(9): 15681575.

9. Posti JP, Takala RSK, Lagerstedt L et all. Correlation of blood biomarkers and biomarker panels with traumatic findings 
on computed tomography after traumatic brain injury. J Neurotrauma. 2019, Apr 5. [Epub ahead of print].

10. Lagerstedt L, Egea-Guerrero JJ, Bustamenate A et all. Combining H-FABP and GFAP increases the capacity to differentiate between CT-positive and CT-negative patients with mild traumatic brain injury. PLoS One. 2018, Jul 9; 13(7):e0200394.
11. Zetterberg $\mathrm{H}$, Smith $\mathrm{DH}$, Blennow K. Biomarkers of mild traumatic brain injury in cerebrospinal fluid and blood. Nat Rev Neurol. 2013 Apr; 9(4): 201-210.

12. Castellani RJ, Perry G. Tau biology, taupathy, traumatic brain injury and diagnostic challenges. J Alzheimers Dis. 2019; 67(2): 447-467. 